\title{
Una aproximación a los Costos Indirectos de la Discapacidad en Colombia
}

\author{
Janeth Hernández-Jaramillo ${ }^{1}$, Iván Hernández-Umaña ${ }^{2}$ \\ ${ }^{1}$ Fonoaudióloga. M. Sc. Discapacidad e Inclusión Social. Universidad Colegio Mayor de \\ Nuestra Señora del Rosario. Facultad de Rehabilitación y Desarrollo Humano. \\ E-mail: janeta@aolpremium.com \\ 2 Economista. M. Sc., Ph. D Economía. Profesor Asociado. Facultad de Ciencias Económicas. \\ Universidad Nacional de Colombia. \\ E-mail: idhernandezu@unal.edu.co
}

Recibido 19 Octubre 2004/Enviado para Modificación 12 Diciembre de 2004/Aceptado 23 Abril 2005

\section{RESUMEN}

Este documento plantea las relaciones entre la política económica y la discapacidad. Representa un intento por proveer un análisis teórico para la política económica en discapacidad en Colombia.

Métodos Se utilizaron los valores absolutos y porcentuales de la distribución de la discapacidad en la población colombiana usando los datos de tres fuentes oficiales de 1981 a 2003. Se seleccionaron las variables: edad, educación, trabajo, estrato socioeconómico, ingresos y dependencia. Se formularon y contrastaron diversas hipótesis con análisis de frecuencias y prueba de $\mathrm{Chi}^{2}$ para estimar diferencias entre grupos.

Resultados El análisis estableció que la vulnerabilidad debida a la discapacidad es diferente según: 1. El género con mayor impacto en hombres; 2. La edad: mayor impacto para los grupos de mayor edad y en los tres últimos años, mayor en el grupo de 25 a 55 años en riesgo potencial de discapacidad; 3. El área: mayor en áreas rurales y, 4. El nivel socioeconómico: las personas en condición de discapacidad (PCD) pertenecen a los estratos más bajos. El perfil de las PCD se caracteriza por tener bajo nivel educativo, percibir y vivir con menos de un salario mínimo mensual, estar desempleadas, ubicarse en los estratos uno y dos, hacer parte de los hogares calificados como pobres, ser varones y vivir en el área rural.

Conclusiones La pobreza parece exacerbar la discapacidad a expensas de la vulnerabilidad, el riesgo y la restricción en las oportunidades para el bienestar. Estos resultados podrían ser usados como argumentos en la reorientación de las políticas y acciones para incrementar el acceso de las PCD a las oportunidades sociales y económicas. 
Palabras Clave: Discapacidad, economía, política de salud. (fuente: DeCS, BIREME)

ABSTRACT

An approach to indirect costs of disability in Colombia

Objectives This document studies the relationships between economic policy and disability, representing an attempt at providing a theoretical analysis for economic policy of disability in Colombia.

Methods The absolute figures and percentages of distribution of disability in the Colombian population from 1981 to 2003, obtained from 3 official sources, were used. The following variables were selected: age, education, employment, socio-economic level, income and dependence. Several hypotheses were formulated and contrasted with frequency analysis and the Chi-square test to estimate differences between groups.

Results The analysis demonstrated that vulnerability to disability is different according to: 1. Sex, with a greater impact on males; 2. Age: greater impact for older age groups and, in the last 3 years, greatest impact in the 25 to 55 year age group under potential risk of disability; 3: Region: greater vulnerability in rural areas and 4. Socio-economic level: persons with disabling conditions belong to the lower socio-economic strata. The typical profile of persons with disabling conditions is poor education, income of less than a minimum monthly wage, unemployed, belonging to the 1 or 2 socio-economic strata, forming part of homes defined as poor, being male and living in rural areas.

Conclusions Poverty seems to enhance disability increasing vulnerability, risk and restricting opportunities for welfare. These results can be used as arguments for redirecting the policies and actions for greater access of disabled persons to social and economic opportunities.

Key Words: Disability, economics, health policy (source: MeSH, NLM)

S egún estimativos de la Organización Mundial de la Salud, un 12 \% del total de habitantes de un país pueden estar en condición de discapacidad. Si la población colombiana fuera de 44 millones, cerca de 5 millones de personas se encuentran en situación de discapacidad. Las PCD -muchos víctimas de la violencia y del conflicto armado- viven en regiones como Centroamérica, concentrados en sectores marginados y empobrecidos, fuera del mercado de trabajo y cuando menos la mitad de ellas en edad productiva (1).

La magnitud y los costos de la discapacidad para América Latina incluida Colombia, distan de ser aproximaciones mensurables de esa realidad. Los problemas en la sistematización de la información sobre discapacidad no permiten conocer su comportamiento espacial ni temporal. De igual forma, 
las diversas concepciones de la discapacidad se refieren a grupos distintos: los términos "discapacitado", "inválido", “incapacitado", "lisiado", "impedido" y "minusválido" suponen valores distintos que hace difícil establecer su magnitud y sus costos.

La discapacidad es un proceso dinámico que fluctúa en extensión y severidad a lo largo de la vida y puede limitar o no la capacidad para trabajar y vivir de forma independiente. Debe ser entendida además, como un fenómeno socioeconómico; los estudios económicos han contribuido sustancialmente a entender asuntos relacionados con la discapacidad.

Los costos en discapacidad no son uniformes para toda la población; se relacionan con la severidad de la limitación funcional y el nivel de discapacidad presentes. Los altos costos de la discapacidad pueden clasificarse como directos e indirectos. Los costos directos incluyen: el cuidado médico y de rehabilitación, las intervenciones tempranas y agresivas, la vigilancia de las condiciones crónicas y el uso de la tecnología. La investigación académica se ha centrado en los costos directos, particularmente en la organización, prestación y financiación de las intervenciones para personas en condición de discapacidad y en medir poblaciones de interés (e.j., condiciones de alta prevalencia e incidencia) (2-5). Los costos indirectos están representados en la pérdida de ganancias resultado de la condición de discapacidad (5) y pueden incluir: la perdida laboral, la disminución de ingresos, los bajos índices de satisfacción y bienestar, los pagos relacionados con el aseguramiento social, las indemnizaciones, los apoyos compensatorios del gobierno y la beneficencia pública, la dependencia que absorbe la familia o el pago de un cuidador y la perdida de consumir menos bienes y servicios, como no pagar impuestos y no asumir otras responsabilidades sociales.

Una revisión sistemática de los estudios sobre los asuntos económicos en discapacidad permite deducir que los costos indirectos son difíciles de estimar (6). No existe información económica sistemática que permita conocer el costo social de la discapacidad Un problema común es que la distribución de las variables de costo está sesgada por el conjunto de derechos del Sistema de Seguridad Social y fuera de él y quizás también por la falta de conciencia y de voluntad política. Los modelos econométricos propuestos no reflejan todas las circunstancias particulares individuales o grupales. Aunque se reconoce que los ingresos y el empleo son solo un aspecto del bienestar, los modelos económicos los usan para medir el bienestar (6). Varios estudios demuestran que las PCD tienen una baja participación en la fuerza de trabajo y presentan los índices de desempleo más altos $(7,8)$. 
En el año 2000, el para entonces Ministerio de Salud, realizó un análisis de la redistribución de beneficios a través del Sistema de Seguridad Social Integral (9). Este estudio permitió definir y clasificar la discapacidad según su severidad, precisar y categorizar el pronóstico funcional, listar y costear las posibles ayudas técnicas requeridas y conocer el comportamiento de la discapacidad y su incidencia por mil habitantes según criterios de severidad y pronóstico. La estimación de los costos fue hecha usando los conjuntos integrados relacionados con la severidad y el pronóstico funcional para cada fase y ciclo completo de rehabilitación funcional.

El Ministerio de Educación y la Pontificia Universidad Javeriana realizaron en 1994 un estudio demográfico en discapacidad. Fueron encuestados 60 037 hogares de nueve ciudades del país. Esta caracterización señaló que el $12,1 \%$ de los menores de 14 años, el 60,3 \% de quienes estaban en el rango de edad entre los 15 y 59 años y el 27,6 \% de los mayores de 60 años de edad tienen necesidades básicas insatisfechas. El rango de edad que presenta mayor discapacidad está entre los 46 y 50 años con un 44,6\%, seguido de los mayores de 60 años. En el estudio no se advirtieron diferencias por género en términos de la magnitud de la discapacidad (10). El mayor porcentaje de PCD corresponde a jefes de hogar con un $39 \%$, de quienes dependen otros para su manutención. Del total de registros, el 23,6 \% está distribuido entre los estratos uno, dos y tres. El desempleo en la población en edad de trabajar alcanza el 39,9 \%. El 55 \% de los hogares reportaron ingresos entre uno y dos salarios mínimos mensuales legales vigentes (SMMLV) y solo el 4,9\% de más de tres salarios mínimos. Únicamente un 7,8 \% de las personas está afiliado al régimen contributivo de Seguridad Social en Salud (10).

Aunque los costos de la disminución del bienestar de las personas en condición de discapacidad no pueden ser medidos con exactitud, es posible estimar en dónde están centrados dichos costos.

El propósito del presente estudio fue realizar una aproximación empírica a los costos indirectos o costos de oportunidad en discapacidad mediante un análisis de datos secundarios provenientes del Departamento Administrativo Nacional de Estadística (DANE).

\section{MÉTODOS}

De tres fuentes de datos oficiales nacionales, se seleccionaron las distribuciones porcentual y absoluta de las variables educación, trabajo, estrato socioeconómico, ingresos y dependencia, de las categorías relacionadas con la 
discapacidad. A partir de ellas se establecieron sus comportamientos en razón del tiempo, la edad, el género y el área (urbana y rural). Se formularon las siguientes hipótesis: 1 . Las PDC se encuentran social y económicamente excluidas de las oportunidades de empleo, ingresos y educación; 2. La discapacidad aumenta con la edad; 3. Existe un aumento de las cifras de discapacidad para grupos atípicos de edad; 4. Las oportunidades de educación son bajas para las PCD; 5. Hay una relación entre pobreza y discapacidad; 6. La discapacidad se concentra en las áreas rurales y 7. La discapacidad se manifiesta de forma distinta en razón del género.

Las fuentes utilizadas fueron: 1. El Registro para la Localización y Caracterización de Personas con Discapacidad (11); 2. La Encuesta de Calidad de Vida 2003 en relación con los hogares con "personas con dificultades permanentes para trabajar (PDP)” (12); y 3. La serie más larga posible de la Encuesta Continua de Hogares de los años 1981 a 2003 en lo referente a la incapacidad permanente para trabajar (IPT) de la población económicamente inactiva (13).

El Registro para la Localización y Caracterización de Personas con Discapacidad es una herramienta técnica que permite recolectar información para conocer y examinar la situación de la población colombiana con discapacidad respecto al grado de satisfacción de las necesidades humanas (11). Incluyó un total de 9135 personas previamente inscritas en la convocatoria de cada municipio.

Para el presente estudio se utilizaron las tablas dinámicas que contienen la totalidad de los registros, lo que permitió realizar nuevos cruces y análisis de variables seleccionadas no incorporados en el análisis primario del DANE. De los datos del Registro (11) se eligieron cinco grupos de edad que constituyen el 65,7 \% por considerarlos potencialmente productivos. Para cada grupo se obtuvieron las distribuciones de frecuencia de las variables seleccionadas por conveniencia de los siguientes módulos: localización y vivienda, identificación personal, caracterización y origen de la discapacidad, salud, educación, participación y trabajo.

La Encuesta de Calidad de Vida es una herramienta que cuantifica y caracteriza las condiciones de vida de los pobres y de los no pobres. De la Encuesta del 2003, se obtuvieron los datos relacionados con los hogares que refirieron tener las PDP y se realizó un análisis cruzado con la variable pobreza. Se tomaron la distribución de frecuencia y los porcentajes de hogares con PDP tanto del total nacional como por regiones y áreas. 
La Encuesta Continua de Hogares mide los cambios en empleo, desempleo y otras variables relacionadas con la fuerza de trabajo (12). La Encuesta incluye variables como la edad, el sexo, el estado civil, la migración, la educación y otras. De la Encuesta se obtuvieron los porcentajes de la población económicamente inactiva con IPT denominados incapacitados. El ejercicio estadístico se realizó con base en los datos correspondientes a los años 1981 al 2003. Se llevaron a cabo análisis separados de los años 1981 a 1999 y de 2001 a 2003 debido a que la Encuesta tiene cambios conceptuales entre esos dos periodos. A partir de las bases de datos, se construyeron tablas con los valores absolutos para la población IPT por año, género y regiones y áreas por corresponder éstas a variables principales de la Encuesta. Para los años 2001 a 2003 se seleccionó la distribución geográfica en las categorías cabecera, rural y total nacional; esa distribución no existe para los años 1981 a 2001 periodo en el cual se dispone de distribución en 11 áreas metropolitanas. Este tratamiento de la Encuesta permitió estimar la proporción de personas con IPT entre los años 1981 y 1999 discriminados por género y grupos etáreos para las 11 áreas metropolitanas. Los años 2001 a 2003 fueron analizados por grupos de edad, sexo y localización total nacional, cabecera y rural como se mencionó.

\section{RESULTADOS}

Se presenta el análisis por cada fuente de datos, debido a que cada una de ellas posee metodología diferente.

1. Registro para la Localización y Caracterización de Personas con Discapacidad

De las condiciones de vida y características principales del total de personas registradas $(n=9135)$ se estableció que el $82 \%$ viven en las cabeceras municipales. Pertenecen a los estratos uno y dos el 82,7 \%. El 48,1 \% no tienen casa propia. La discapacidad afecta igual a hombres y mujeres. El 19,6 $\%$ son menores de edad, un 51,7 \% están entre los 18 y 64 años y el 28,5 \% restante lo constituyen los mayores de 65. Del total de personas registradas un 29,6 \% tiene personas a su cargo. La mayor concentración de personas estuvo en las cabeceras. El grupo de 18 a 74 años tuvo el mismo comportamiento espacial que el total.

Para quienes conocían el origen de su discapacidad, la distribución de frecuencias por grupos de edad mostró que los traumatismos, incluida la violencia, tienen un peso equiparable con las condiciones adversas del desarrollo, con un 22 y un $20 \%$, respectivamente. Del total de personas entre los 
18 y 74 años de edad el 42 \% calificaron su condición como resultado de enfermedad general. La enfermedad profesional solo representó un 3,2 \%.

Los porcentajes más altos de PCD pertenecen a los estratos uno y dos, con un 39 y un $42 \%$, respectivamente. Sólo un 1,6 \% de las personas registradas están en el estrato cuatro. Tan solo un $19 \%$ de las PCD entre los 18 y 64 años de edad respondieron estar trabajando. Del mismo grupo, el 92,4 \% reciben uno o menos de un SMMLV. El restante $81 \%$ no participa del trabajo; asumiendo un ingreso equivalente a un SMMLV para este grupo, la pérdida absoluta representaría cerca de 20500 millones de pesos por año, una perdida aproximada de 15 SMMLV por persona al año.

Si se toma el rango de edad de 20 a 64 años ( $\mathrm{n}=4$ 727), (potencialmente productivos y sin edad de pensionarse), el $72 \%$ se encuentran incapacitados para trabajar y tan solo el 1,5 \% reciben pensión. En costos, asumiendo una pensión igual a un SMMLV, representa cerca de 300 millones de pesos por año. La conversión señala la pérdida de 0,2 SMMLV por persona al año.

En relación con la actividad de los últimos seis meses y el ingreso percibido (pensión, jubilación, trabajo o renta) el 79,6 \% no realiza una actividad que genere ingresos. El restante 19,4 \% está buscando trabajo, estudiando, son incapacitados para trabajar sin pensión, realizan oficios del hogar u otra no especificada.

En el rango de edad de 18 a 74 años el 92,6 \% recibe uno o menos de un SMMLV. El 1,6 \% de los encuestados informó que recibe dos SMMLV y porcentajes por debajo de $1 \%$ tres o más SMMLV. De allí que casi todas las personas viven de un SMMLV.

El 37,7 \% de los discapacitados entre los 18 y los 74 años de edad refieren tener personas a su cargo. De ellas un 98,2 \% recibe uno o menos de un SMMLV. Se deduce que cerca de 4530 personas viven con alrededor de 175 mil pesos mensuales al suponer que una sola persona es dependiente en su manutención. Si las personas a cargo son dos esto significa que tres personas, incluida la cabeza del hogar, viven con 116 mil pesos mensuales. Estas estimaciones sugieren que un porcentaje importante de personas se encuentran por debajo de la línea de pobreza en tanto viven con menos de un SMMLV. El 80,3\% de los discapacitados del rango de edad de 18 a 74 años respondieron no estar participando en actividades económicas.

En el rango de edad seleccionado tan solo el 5,3\% aprobaron el $11^{\circ}$ de educación básica, de tal forma que del Registro el 90 \% no tiene educación 
básica secundaria. En el rango de edad de 18-74 años, el 40,1 \% ( $n=2$ 411) requieren permanente ayuda de otra persona. Esto se traduce en la absorción de una persona potencialmente productiva y si una vez más, se considerara que esa persona podría devengar cuando menos un SMMLV, el monto representaría 10126 millones de pesos por año. En pérdida por persona al año esto es igual a 12 SMMLV.

Los adultos mayores (65-74 años) requieren ayuda permanente en un porcentaje mayor (61 \%) que el grupo de jóvenes (18-19), para quienes el porcentaje es igual a 39. Esto supone una mayor limitación funcional para los adultos mayores y equivaldría a 3300 millones de pesos. Al año esto son 11 SMMLV por persona. De quienes requieren de ayuda permanente, el 2,6 $\%$ tienen un cuidador externo empleado. En el mismo rango de edad, un $29,4 \%$ de quienes requieren ayuda permanente, a su vez tienen personas a su cargo.

Del total de personas que demandan ayuda permanente, el $62 \%$ sufren alteración del sistema nervioso, aspecto de gran interés tanto por la causa como por las consecuencias en términos de la discapacidad.

Del total de personas de este Registro, el 29\% carece de aseguramiento en el Sistema General de Seguridad Social en Salud. El 90,6 \% no recibe servicios de rehabilitación y de ellos el 77,5 \% refieren como razones para no asistir a rehabilitación la falta de dinero y los problemas de accesibilidad.

En general, el Registro señala que las PCD: 1. Viven con menos de un SMMLV; 2. Casi todos pertenecen a los estratos uno y dos; 3. El $40 \%$ tiene personas a cargo; 4. No tienen trabajo; 5. Presentan los niveles de educación bajos (no tiene secundaria completa); 6. Necesitan rehabilitación para el trabajo; 7. Presentan deficiencias asociadas a enfermedad general; 8. No requieren ayuda permanente, cuando menos los grupos en edad de trabajar; 9. La mayoría tienen afiliación en salud.

\section{Encuesta de Calidad de Vida}

De un total de 11194108 hogares, un 11,7 \% corresponde a hogares con PDP ( $n=1310458$ ) y de ellos un 19,5 \% son clasificados como hogares pobres ( $\mathrm{n}=255686)$.

La proporción de discapacitados entre los pobres es el doble del de la población total. La diferencia en la proporción de personas con dificultades permanentes entre pobres y no pobres es estadísticamente significativa 
(prueba de $\mathrm{Chi}^{2} \mathrm{p}=0,006$ ). Un cálculo del riesgo relativo de discapacidad si se clasifica como pobre, revela que el riesgo relativo (RR) es 1,2 veces mayor que si no se clasifica como tal (RR para discapacidad si se clasifica como pobre 0,113 , RR si no se clasifica como pobre 0,136 ). De manera comparativa, un cálculo de riesgo relativo para la población rural solamente, revela un RR 2,07 veces mayor. El riesgo relativo de discapacidad si se clasifica como pobre es mayor que si no se califica como tal.

\section{Análisis de la Encuesta Continua de Hogares}

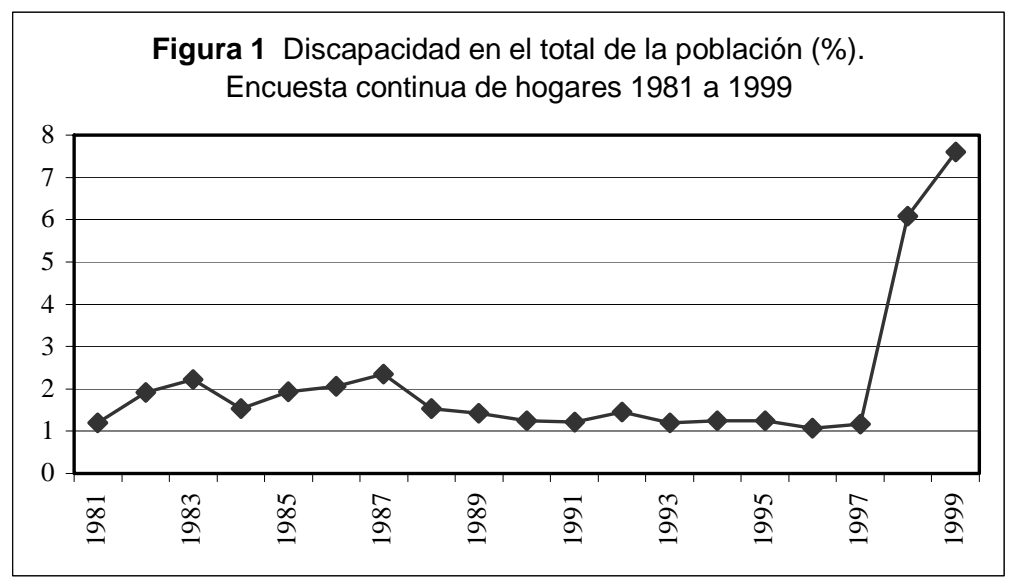

El análisis para el periodo 1981-1999 mostró que el porcentaje de personas con IPT para la población total permaneció estable, con una fluctuación en razón del tiempo entre el 1 y el 2 \% hasta los años 1998 y 1999 a partir de los cuales se registra un incremento significativo (Figura 1).

La Figura 2 compara el promedio de los porcentajes de IPT de los años 1981 a 1996 con el de los años 1997 a 1999, por grupos de edad, e ilustra la diferencia entre los dos periodos. Se aprecia un incremento porcentual de personas con IPT para los últimos dos años del periodo. Este incremento ocurre a expensas de todos los grupos etáreos con mayor representación para los grupos de mayor edad (60 a 79; 80 y más años). El inesperado incremento porcentual en los años 1997 a 1999 podría explicarse por la crisis económica que tuvo lugar en 1997. Cuando en estos mismos periodos se estiman las diferencias por género se observa una marcada heterogeneidad, con 
porcentajes de IPT diez veces mayores para los hombres, aunque sugiere algún tipo de sesgo muestral.

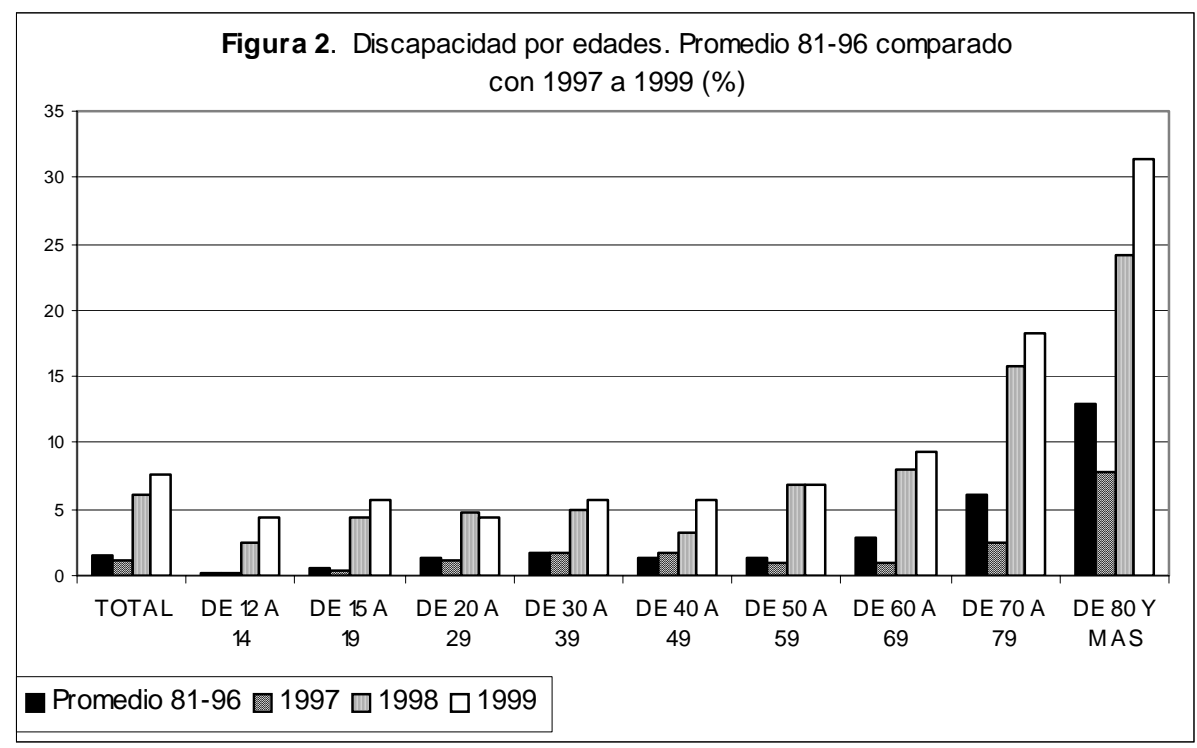

Para los años 2001 a 2003 los porcentajes de las personas con IPT señalaron diferencias en la distribución por áreas y género, siendo mayor el porcentaje para los hombres y en el área rural, en todos los grupos de edad. Se observa consistencia en el incremento porcentual en los años 1998 y 1999 el cual reaparece en el 2001 y decrece en los años 2002 y 2003.

En general, la Encuesta Continua de Hogares permite establecer que: 1. Hay un mayor porcentaje de hombres con IPT que de mujeres; 2. Los grupos de edad en los cuales se concentra el mayor porcentaje de IPT son los de edad avanzada (70 y más); 3. Existe una concentración mayor de IPT en el área rural; 4. Existe un incremento porcentual de IPT en los años 1998 y 1999, válido para todos los grupos de edad de manera proporcional; y 5. La distribución de hogares pobres con IPT permanece estable a lo largo de los años.

\section{DISCUSIÓN}

Se reconoce que las fuentes de información estadística aquí empleadas podrían advertir problemas en la conceptualización de la discapacidad y en 
la forma en como ésta ha sido medida, además de los sesgos de índole metodológico y estadístico que se le han atribuido a las encuestas nacionales. No obstante, estas fuentes constituyen la mejor información disponible para Colombia. La mayor parte de las estimaciones sobre la discapacidad en América Latina al igual que en el ejercicio presente, provienen de los censos poblacionales y de encuestas de hogares (1). Las diferencias metodológicas de estas fuentes de información estadística, que obedecen a propósitos distintos en su formulación y administración, no permiten realizar comparaciones entre los datos.

Aunque se reconoce que los ingresos, el empleo y la educación son solo un aspecto del bienestar, el presente análisis usa estas variables como una aproximación a la exclusión económica, en la medida en que se entiende su capacidad de estimar los niveles de bienestar mejor de lo que lo harían otros satisfactores. Además éstos han sido usados como indicadores de efectividad de la política pública.

Como lo mostró el análisis de los datos proporcionados por el Registro las PCD están concentradas en los estratos uno y dos; lo cual pone de manifiesto su reducida oportunidad de movilización en la estructura social. El hecho de que tan solo el $5 \%$ de las personas en condición de discapacidad alcanzaran el nivel de educación secundaria, sugiere una pobre oportunidad de formación y con ello un rango estrecho de cualificación laboral.

Como se mostró en el análisis, casi el 30 \% de las PCD no tienen protección social en salud y por tanto no reciben rehabilitación. A ello se le suma que un alto porcentaje de quienes no asisten a rehabilitación, refieren como razones la inaccesibilidad a los servicios. En contraste, el hecho de que cerca del $70 \%$ de las personas del Registro tengan aseguramiento en salud supone una serie de beneficios que deberían aplicar para el total de las PCD.

El desempleo que afecta a cerca del $81 \%$ de las PCD supone una pérdida productiva inmensurable para el país; en tanto que ello implica no alcanzar otros satisfactores relacionados con el empleo. La pensión por incapacidad laboral debe ser considerada otra de las dimensiones de los costos indirectos ya que representa un pago a una persona en edad productiva y supone un gasto para el Sistema de Seguridad Social.

La dependencia de una PCD conlleva que otro miembro de la familia vea reducida su participación laboral. De igual forma una PCD que no puede trabajar reduce la renta total potencial de la familia, forzando a los otros miembros a trabajar más. Cuando la PCD es cabeza de familia, su situación 
ocupacional tiene un impacto negativo en el índice de participación del resto de la familia; esto es, las oportunidades del hogar se ven restringidas.

En el análisis estadístico de la Encuesta Continua de Hogares se reconoce que el ítem IPT no incluye el total de PCD, pero resulta válido en la identificación de aquellas personas que debido a una condición de salud o funcionamiento permanente no pueden participar de la producción de bienes y servicios. Esta Encuesta señala cambios porcentuales en las personas con IPT que quizás puedan explicarse por la propia composición demográfica, los niveles de educación, la naturaleza cambiante del empleo, el incremento de la discapacidad, la caracterización de tipo y severidad de la discapacidad, los cambios en las políticas de beneficios en el Sistema de Seguridad Social y las condiciones de violencia inherentes a países como Colombia. Además, otro aspecto que podría afectar la participación de las PCD en la fuerza laboral es la reducción en la esperanza de vida: cuanto más corta es la expectativa de vida hoy, menor será la proporción de individuos en edad de trabajar y mayores los costos adicionales que la discapacidad genere, tales como los servicios de rehabilitación, la dependencia y las oportunidades para el trabajo.

No obstante el análisis presentado sobre lo que representa en costos la IPT, el efecto de la discapacidad sobre la participación en el trabajo es incierto, en la medida en que los cálculos dependen de los diferentes escenarios; es decir, de la perdida o disminución de la productividad o de la perdida o menor oportunidad de empleo para las PCD.

Los accidentes, la violencia y los conflictos son una fuente importante de discapacidad. Para el presente análisis los traumatismos alcanzan el $20 \%$ del origen de la discapacidad. Sin embargo, contrario a lo presupuestado, esta cifra tiene el mismo peso que las causas relacionadas con enfermedades generales y del desarrollo.

Los datos presentados sugieren que la discapacidad afecta más a los hombres. Esto quizás este relacionado con un mayor riesgo por el tipo de actividades laborales que realizan los hombres, su mayor participación en el conflicto armado y la exposición a factores de riesgo relacionados con los hábitos de vida y de consumo. La mayor concentración de PCD parece estar en el área rural, ello debido posiblemente a las condiciones de salubridad, la inequidad en el acceso a los servicios de salud, las menores oportunidades de cualificación laboral y al conflicto armado. 
A partir de la Encuesta de Calidad de Vida la discapacidad constituye un factor asociado a la pobreza, sin poder colegir para Colombia la relación de causalidad entre tales variables, aunque varios estudios señalan también que las condiciones de pobreza aumentan el riesgo de discapacidad (14).

Los datos ponen de manifiesto que las PCD tienen los niveles más bajos de educación, de ingresos, de capacidad de ahorro y de otros activos en comparación con el resto de la población. Ello soporta la noción de exclusión económica de las PCD. El aumento de la discapacidad en razón de la edad, se sustenta en una distribución porcentual mayor para los grupos de edad avanzada. Los datos no permiten sostener que en la última década hayan aumentado los porcentajes de discapacidad en razón de la violencia para grupos atípicos de edad, por ejemplo para los más jóvenes. No obstante, el ejercicio estadístico de los años 2001 a 2003 reveló un incremento porcentual para el grupo de 25 a 55 años, no siendo posible establecer que ello fuera a expensas de la violencia. Por contraste, el claro predominio de discapacidad en la edad avanzada, señala la insoslayable importancia de las enfermedades crónicas en su origen. Tal como se mencionó, las oportunidades de educación son bajas para las personas en condición de discapacidad. Hay diferencias marcadas en la distribución por cabecera y rural, con mayores porcentajes para esta última. Existe un porcentaje mayor de hombres en condición de discapacidad. Finalmente, el perfil de las PCD parece caracterizarse por tener niveles de educación bajos, percibir y vivir con menos de un SMMLV, estar desempleadas, estar en los estratos uno y dos, pertenecer a los hogares calificados como pobres, ser hombres y vivir en el área rural.

Es posible señalar que la exclusión económica, reduce las oportunidades para que las PCD contribuyan a su hogar y a su comunidad y aumenta el riesgo de caer en la pobreza. Las consecuencias económicas de la discapacidad han sido subestimadas en los análisis económicos y desconocidas por los hacedores de política pública. El análisis presentado ilustra la exclusión económica de las PCD y con ello la inobservancia de sus derechos. Aunque el presente estudio no permitió conocer los costos indirectos de la discapacidad, quizás logre una aproximación de las dimensiones que concentran la perdida de oportunidades para el bienestar, ello hace posible establecer las prioridades en la política pública en discapacidad. De cualquier forma la política económica en discapacidad deberá considerar la cobertura de la rehabilitación laboral y de la educación, por cuanto estas dos dimensiones resultan definitivas para la participación en las actividades económicas del país.

Los resultados del presente análisis tienen desde luego limitaciones y deberán ser leídos teniendo en consideración las restricciones que suponen la 
metodología de muestreo utilizada por las fuentes de información primaria, lo insuficiente de las variables incorporadas en las encuestas, la propia definición y operacionalización de las variables, las dificultades en el acceso a la información estadística nacional, la propia calidad de los estudios que supone sesgos en los datos y la consistencia de la información a partir de fuentes distintas.

Investigaciones futuras deberán realizar el análisis de los ingresos de las personas que en la Encuesta Continua de Hogares aparecen con IPT, dado que para el presente estudio dicha información no estuvo disponible y su incorporación como variable econométrica permitiría aportar evidencia sobre los costos indirectos de la discapacidad. De igual forma deberán ser incorporadas otras variables de las fuentes de datos empleadas que no fueron incluidas en el presente análisis y que podrían resultar determinantes en la argumentación política

\section{REFERENCIAS}

1. Inter American Development Bank. Disability and the labor market in American Latin. [Internet]. Disponible en: www.iadb.org. Consultado: Septiembre 2003.

2. Walls RT. Measurement of Client Outcomes in Rehabilitation. In: Handbook of measurement and evaluation in rehabilitation. Gaithersburg MD: Aspen; 2001. p. 311-337.

3. Cuervo C, Escobar M, Trujillo A. Factores que determinan el impacto de los servicios de rehabilitación fisioterapéutica, ocupacional y fonoaudiológica en las instituciones de la Secretaría Distrital de Salud de Santa Fe de Bogotá; 1998. [Internet]. Disponible en: www.saludcapital.gov.co. Consultado Octubre 2003.

4. WHO. Cost Analysis for Management of Rehabilitation Programmers. Rehabilitation Unit. Division of Health Organization; 1997. [Internet]. Disponible en: www.who.org. Consultado Septiembre 2003.

5. Institute of Medicine. Economic Issues and the Cost of Disability. Pain and disability: Clinical Behavioral and Policy Perspectives; 1987. p. 87-100. [Internet]. Disponible en: http: //www.nap.edu/openbook. Consultado Agosto 2003.

6. Institute of Medicine. Enabling America. Assessing the role of Rehabilitation Science and Engineering. Washington: National Academy Press; 1997.

7. Russell M. Disablement, Oppression and Political Economy. Journal of Disability Policy Studies 2001; 12: 87-95.

8. Russell M, Malhotra R. Disability and capitalism. In: Leys C, Panitch L, eds. Socialist Register Suffolk: Merlin Press; 2002. p. 211-228. 
9. Ministerio de Salud. Ocupación, discapacidad y costos: la redistribución de beneficios a través del Sistema de Seguridad Social Integral. Santa Fe de Bogotá DC, Colombia; 2000.

10. Ministerio de Educación. Sistema de Información de Discapacidad. Bogotá DC, Colombia; 1995.

11. Departamento Administrativo Nacional de Estadística-Ministerio de Educación Nacional. Registro para la localización y caracterización de la población con Discapacidad. Bogotá DC, Colombia; 2002

12. Departamento Administrativo Nacional de Estadística. Resumen Metodológico Encuesta Nacional de Hogares. División de Calidad e Interventoría Estadística. Bogotá DC, Colombia; 1998.

13. Departamento Administrativo Nacional de Estadística. Instructivo para el diligenciamiento del formulario de Registro para la Localización y Caracterización de la Población con Discapacidad. Registros Demográficos-Discapacidad. Bogotá DC, Colombia. 2003.

14. Elwan A. Poverty and Disability, a Survey of the Literature; 1999 [Internet] Disponible en: http://www.worldbank.org. Consultado Marzo 2003. 